Supporting information for

\title{
Structure and Conformational Response of Pure and Lithium- Doped Ionic Liquids to Pressure Alterations From Molecular Dynamics Simulations
}

\section{Th. Dhileep N. Reddy and Bhabani S. Mallik*}

Department of Chemistry, Indian Institute of Technology Hyderabad, Kandi-502285, Sangareddy, Telangana, India

Table S1: Non-bonding force field parameters for Pyr13 ion.

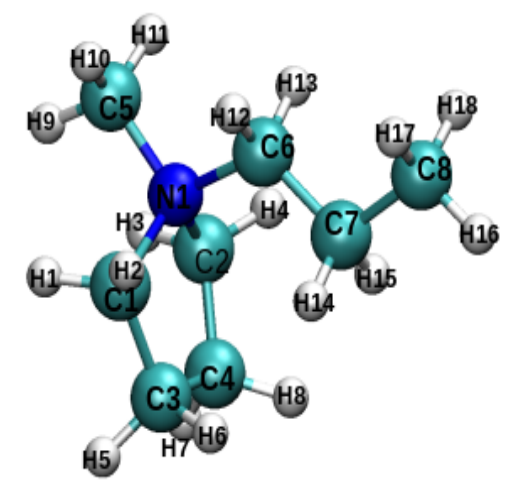

\begin{tabular}{|c|c|c|c|c|}
\hline System & Atom & $q(e)$ & $\sigma(\AA)$ & $\varepsilon(\mathrm{kcal} / \mathrm{mol})$ \\
\hline \multirow[t]{17}{*}{ Pyr $_{13}$} & $\mathrm{~N}_{1}$ & 0.0619 & 3.25 & 0.0625 \\
\hline & $\mathrm{C}_{1}$ & -0.0242 & 3.50 & 0.0660 \\
\hline & $\mathrm{C}_{2}$ & -0.0242 & 3.50 & 0.0660 \\
\hline & $\mathrm{C}_{3}$ & -0.0231 & 3.50 & 0.0660 \\
\hline & $\mathrm{C}_{4}$ & -0.0231 & 3.50 & 0.0660 \\
\hline & $\mathrm{C}_{5}$ & -0.3480 & 3.50 & 0.0660 \\
\hline & $\mathrm{C}_{6}$ & -0.1877 & 3.50 & 0.0660 \\
\hline & $\mathrm{H}_{1}$ & 0.0819 & 2.50 & 0.0300 \\
\hline & $\mathrm{H}_{2}$ & 0.0819 & 2.50 & 0.0300 \\
\hline & $\mathrm{H}_{3}$ & 0.0819 & 2.50 & 0.0300 \\
\hline & $\mathrm{H}_{4}$ & 0.0819 & 2.50 & 0.0300 \\
\hline & $\mathrm{H}_{5}$ & 0.0471 & 2.50 & 0.0300 \\
\hline & $\mathrm{H}_{6}$ & 0.0471 & 2.50 & 0.0300 \\
\hline & $\mathrm{H}_{7}$ & 0.0471 & 2.50 & 0.0300 \\
\hline & $\mathrm{H}_{8}$ & 0.0471 & 2.50 & 0.0300 \\
\hline & $\mathrm{H}_{9}$ & 0.1552 & 2.50 & 0.0300 \\
\hline & $\mathrm{H}_{10}$ & 0.1552 & 2.50 & 0.0300 \\
\hline
\end{tabular}




\begin{tabular}{|c|c|c|c|c|}
\hline \multirow{7}{*}{} & $\mathrm{H}_{11}$ & 0.1552 & 2.50 & 0.0300 \\
\cline { 2 - 5 } & $\mathrm{H}_{12}$ & 0.1109 & 2.50 & 0.0300 \\
\cline { 2 - 5 } & $\mathrm{H}_{13}$ & 0.1109 & 2.50 & 0.0300 \\
\cline { 2 - 5 } & $\mathrm{C}_{7}$ & 0.1172 & 3.50 & 0.0660 \\
\cline { 2 - 5 } & $\mathrm{H}_{14}$ & 0.0228 & 2.50 & 0.0300 \\
\cline { 2 - 5 } & $\mathrm{H}_{15}$ & 0.0228 & 2.50 & 0.0300 \\
\cline { 2 - 5 } & $\mathrm{C}_{8}$ & -0.2411 & 3.50 & 0.0660 \\
\cline { 2 - 5 } & $\mathrm{H}_{16}$ & 0.0812 & 2.50 & 0.0300 \\
\cline { 2 - 5 } & $\mathrm{H}_{17}$ & 0.0812 & 2.50 & 0.0300 \\
\cline { 2 - 5 } & $\mathrm{H}_{18}$ & 0.0812 & 2.50 & 0.0300 \\
\hline
\end{tabular}

Table S2: Non-bonding force field parameters for $\mathbf{P y r}_{16}$ ion.

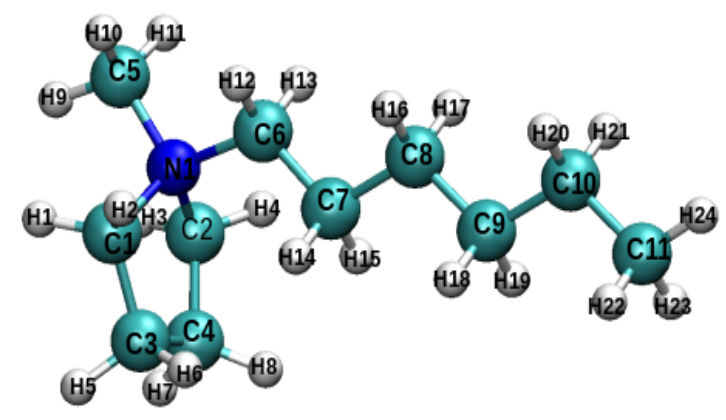

\begin{tabular}{|c|c|c|c|c|}
\hline System & Atom & $\mathrm{q}(\mathrm{e})$ & $\sigma(\AA)$ & $\varepsilon(\mathrm{kcal} / \mathrm{mol})$ \\
\hline Pyr 16 & $\mathrm{~N}_{1}$ & 0.1005 & 3.25 & 0.0625 \\
\cline { 2 - 5 } & $\mathrm{C}_{1}$ & -0.0229 & 3.50 & 0.0660 \\
\cline { 2 - 5 } & $\mathrm{C}_{2}$ & -0.0229 & 3.50 & 0.0660 \\
\cline { 2 - 5 } & $\mathrm{C}_{3}$ & -0.0311 & 3.50 & 0.0660 \\
\cline { 2 - 5 } & $\mathrm{C}_{4}$ & -0.0311 & 3.50 & 0.0660 \\
\cline { 2 - 5 } & $\mathrm{C}_{5}$ & -0.3605 & 3.50 & 0.0660 \\
\cline { 2 - 5 } & $\mathrm{C}_{6}$ & -0.1683 & 3.50 & 0.0660 \\
\cline { 2 - 5 } & $\mathrm{H}_{1}$ & 0.0784 & 2.50 & 0.0300 \\
\cline { 2 - 5 } & $\mathrm{H}_{2}$ & 0.0784 & 2.50 & 0.0300 \\
\cline { 2 - 5 } & $\mathrm{H}_{3}$ & 0.0784 & 2.50 & 0.0300 \\
\cline { 2 - 5 } & $\mathrm{H}_{4}$ & 0.0784 & 2.50 & 0.0300 \\
\cline { 2 - 5 } & $\mathrm{H}_{5}$ & 0.0487 & 2.50 & 0.0300 \\
\cline { 2 - 5 } & $\mathrm{H}_{6}$ & 0.0487 & 2.50 & 0.0300 \\
\cline { 2 - 5 } & $\mathrm{H}_{7}$ & 0.0487 & 2.50 & 0.0300 \\
\hline
\end{tabular}




\begin{tabular}{|c|c|c|c|c|}
\hline \multirow{1}{*}{} & $\mathrm{H}_{8}$ & 0.0487 & 2.50 & 0.0300 \\
\cline { 2 - 5 } & $\mathrm{H}_{9}$ & 0.1547 & 2.50 & 0.0300 \\
\cline { 2 - 5 } & $\mathrm{H}_{10}$ & 0.1547 & 2.50 & 0.0300 \\
\cline { 2 - 5 } & $\mathrm{H}_{11}$ & 0.1547 & 2.50 & 0.0300 \\
\cline { 2 - 4 } & $\mathrm{H}_{12}$ & 0.0981 & 2.50 & 0.0300 \\
\hline $\mathrm{H}_{13}$ & 0.0981 & 2.50 & 0.0300 \\
\hline $\mathrm{C}_{7}$ & 0.0968 & 3.50 & 0.0660 \\
\hline $\mathrm{H}_{14}$ & 0.0098 & 2.50 & 0.0300 \\
\hline $\mathrm{H}_{15}$ & 0.0098 & 2.50 & 0.0300 \\
\hline $\mathrm{C}_{8}$ & -0.0299 & 3.50 & 0.0660 \\
\hline $\mathrm{H}_{16}$ & 0.0140 & 2.50 & 0.0300 \\
\hline $\mathrm{H}_{17}$ & 0.0140 & 2.50 & 0.0300 \\
\hline $\mathrm{C}_{9}$ & -0.0242 & 3.50 & 0.0660 \\
\hline $\mathrm{H}_{18}$ & 0.0042 & 2.50 & 0.0300 \\
\hline $\mathrm{H}_{19}$ & 0.0042 & 2.50 & 0.0300 \\
\hline $\mathrm{C}_{10}$ & 0.1337 & 3.50 & 0.0660 \\
\hline $\mathrm{H}_{20}$ & -0.0138 & 2.50 & 0.0300 \\
\hline $\mathrm{H}_{21}$ & -0.0138 & 2.50 & 0.0300 \\
\hline $\mathrm{C}_{11}$ & -0.1895 & 3.50 & 0.0660 \\
\hline $\mathrm{H}_{22}$ & 0.0508 & 2.50 & 0.0300 \\
\hline $\mathrm{H}_{23}$ & 0.0508 & 2.50 & 0.0300 \\
\hline $\mathrm{H}_{24}$ & 0.0508 & 2.50 & 0.0300 \\
\hline
\end{tabular}

Table S3: Non-bonding force field parameters for $\mathbf{P y r}_{19}$ ion.

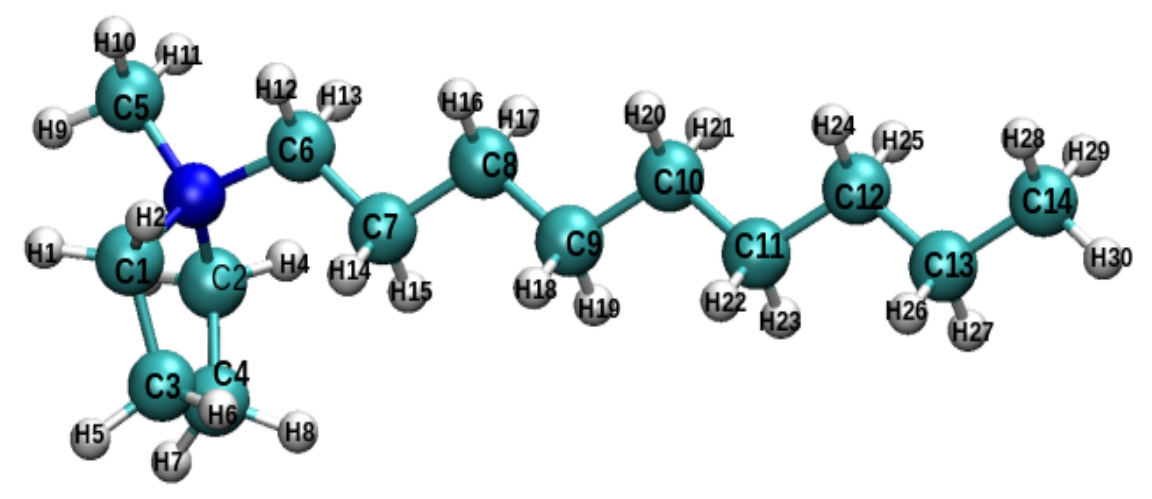

\begin{tabular}{|c|c|c|c|c|}
\hline System & Atom & $\mathrm{q}(\mathrm{e})$ & $\sigma(\AA)$ & $\varepsilon(\mathrm{kcal} / \mathrm{mol})$ \\
\hline Pyr $_{19}$ & $\mathrm{~N}_{1}$ & 0.1078 & 3.25 & 0.0625 \\
\cline { 2 - 5 } & $\mathrm{C}_{1}$ & -0.0268 & 3.50 & 0.0660 \\
\cline { 2 - 5 } & $\mathrm{C}_{2}$ & -0.0268 & 3.50 & 0.0660 \\
\hline
\end{tabular}




\begin{tabular}{|c|c|c|c|c|}
\hline \multirow{1}{*}{} & $\mathrm{C}_{3}$ & -0.0276 & 3.50 & 0.0660 \\
\hline $\mathrm{C}_{4}$ & -0.0276 & 3.50 & 0.0660 \\
\hline $\mathrm{C}_{5}$ & -0.3675 & 3.50 & 0.0660 \\
\hline $\mathrm{C}_{6}$ & -0.1584 & 3.50 & 0.0660 \\
\hline $\mathrm{H}_{1}$ & 0.0788 & 2.50 & 0.0300 \\
\hline $\mathrm{H}_{2}$ & 0.0788 & 2.50 & 0.0300 \\
\hline $\mathrm{H}_{3}$ & 0.0788 & 2.50 & 0.0300 \\
\hline $\mathrm{H}_{4}$ & 0.0788 & 2.50 & 0.0300 \\
\hline $\mathrm{H}_{5}$ & 0.0475 & 2.50 & 0.0300 \\
\hline $\mathrm{H}_{6}$ & 0.0475 & 2.50 & 0.0300 \\
\hline $\mathrm{H}_{7}$ & 0.0475 & 2.50 & 0.0300 \\
\hline $\mathrm{H}_{8}$ & 0.0475 & 2.50 & 0.0300 \\
\hline $\mathrm{H}_{9}$ & 0.1560 & 2.50 & 0.0300 \\
\hline $\mathrm{H}_{10}$ & 0.1560 & 2.50 & 0.0300 \\
\hline $\mathrm{H}_{11}$ & 0.1560 & 2.50 & 0.0300 \\
\hline $\mathrm{H}_{12}$ & 0.0957 & 2.50 & 0.0300 \\
\hline $\mathrm{H}_{13}$ & 0.0957 & 2.50 & 0.0300 \\
\hline $\mathrm{C}_{7}$ & 0.0647 & 3.50 & 0.0660 \\
\hline $\mathrm{H}_{14}$ & 0.0165 & 2.50 & 0.0300 \\
\hline $\mathrm{H}_{15}$ & 0.0165 & 2.50 & 0.0300 \\
\hline $\mathrm{C}_{8}$ & -0.0154 & 3.50 & 0.0660 \\
\hline $\mathrm{H}_{16}$ & 0.0138 & 2.50 & 0.0300 \\
\hline $\mathrm{H}_{17}$ & 0.0138 & 2.50 & 0.0300 \\
\hline $\mathrm{C}_{9}$ & -0.0021 & 3.50 & 0.0660 \\
\hline $\mathrm{H}_{18}$ & -0.0038 & 2.50 & 0.0300 \\
\hline $\mathrm{H}_{19}$ & -0.0038 & 2.50 & 0.0300 \\
\hline $\mathrm{C}_{10}$ & 0.0925 & 3.50 & 0.0660 \\
\hline $\mathrm{H}_{20}$ & -0.0211 & 2.50 & 0.0300 \\
\hline $\mathrm{H}_{21}$ & -0.0211 & 2.50 & 0.0300 \\
\hline $\mathrm{C}_{11}$ & 0.0019 & 3.50 & 0.0660 \\
\hline $\mathrm{H}_{22}$ & -0.0088 & 2.50 & 0.0300 \\
\hline $\mathrm{H}_{23}$ & -0.0088 & 2.50 & 0.0300 \\
\hline $\mathrm{C}_{12}$ & -0.0040 & 3.50 & 0.0660 \\
\hline $\mathrm{H}_{24}$ & -0.0033 & 2.50 & 0.0300 \\
\hline $\mathrm{H}_{25}$ & -0.0033 & 2.50 & 0.0300 \\
\hline $\mathrm{C}_{13}$ & 0.1291 & 3.50 & 0.0660 \\
\hline $\mathrm{H}_{26}$ & -0.0203 & 2.50 & 0.0300 \\
\hline $\mathrm{H}_{27}$ & -0.0203 & 2.50 & 0.0300 \\
\hline $\mathrm{C}_{14}$ & -0.1794 & 3.50 & 0.0660 \\
\hline $\mathrm{H}_{28}$ & 0.0430 & 2.50 & 0.0300 \\
\hline $\mathrm{H}_{29}$ & 0.430 & 2.50 & 0.0300 \\
\hline $\mathrm{H}_{30}$ & 0.430 & 2.50 & 0.0300 \\
\hline & & & \\
\hline 167
\end{tabular}


Table S4: Non-bonding force field parameters for TFSI ion.

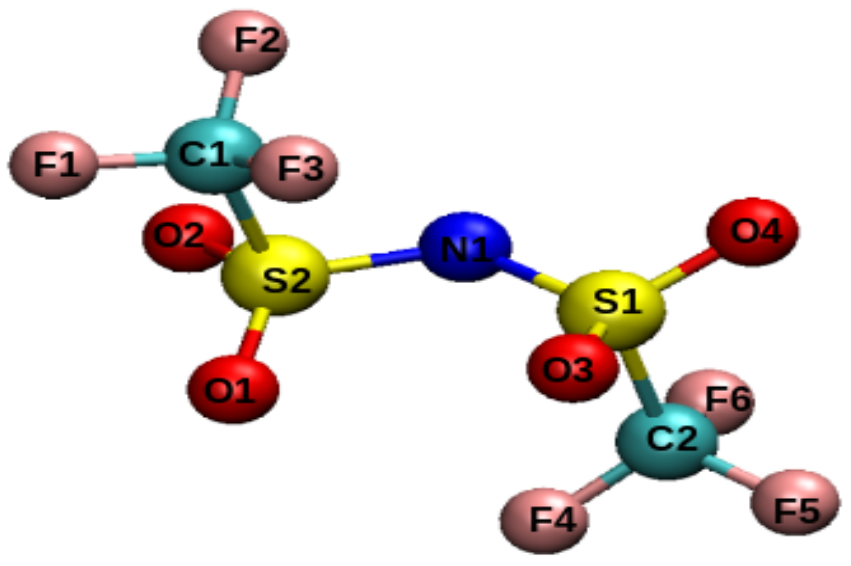

\begin{tabular}{|c|c|c|c|c|}
\hline Ion & Atom & $\mathrm{q}(\mathrm{e})$ & $\sigma(\AA)$ & $\varepsilon(\mathrm{kcal} / \mathrm{mol})$ \\
\hline TFSI & $\mathrm{N}_{1}$ & -0.5111 & 3.25 & 0.1699 \\
\hline & $\mathrm{S}_{1}$ & 0.7986 & 3.55 & 0.2499 \\
\hline & $\mathrm{S}_{2}$ & 0.7986 & 3.55 & 0.2499 \\
\hline & $\mathrm{O}_{1}$ & -0.4232 & 2.96 & 0.2100 \\
\hline & $\mathrm{O}_{2}$ & -0.4232 & 2.96 & 0.2100 \\
\hline & $\mathrm{O}_{3}$ & -0.4232 & 2.96 & 0.2100 \\
\hline & $\mathrm{O}_{4}$ & -0.4232 & 2.96 & 0.2100 \\
\hline & $\mathrm{C}_{1}$ & 0.3064 & 3.50 & 0.0660 \\
\hline & $\mathrm{C}_{2}$ & 0.3064 & 3.50 & 0.0660 \\
\hline & $\mathrm{F}_{1}$ & -0.1344 & 2.95 & 0.0530 \\
\hline & $\mathrm{F}_{2}$ & -0.1344 & 2.95 & 0.0530 \\
\hline & $\mathrm{F}_{3}$ & -0.1344 & 2.95 & 0.0530 \\
\hline & $\mathrm{F}_{4}$ & -0.1344 & 2.95 & 0.0530 \\
\hline & $\mathrm{F}_{5}$ & -0.1344 & 2.95 & 0.0530 \\
\hline & $\mathrm{F}_{6}$ & -0.1344 & 2.95 & 0.0530 \\
\hline
\end{tabular}


Table S5: Density of various systems

\begin{tabular}{|c|c|c|c|c|c|}
\hline \multirow[t]{2}{*}{ 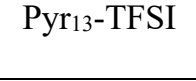 } & Pressure $(\mathrm{GPa})$ & 0.0 & 0.5 & 2.3 & 5.5 \\
\hline & Density $\left(\mathrm{g} . \mathrm{cm}^{-3}\right)$ & 1.411 & 1.436 & 1.504 & 1.581 \\
\hline \multirow{2}{*}{$\begin{array}{c}\operatorname{Pyr}_{13} \text {-TFSI }+ \\
\text { Li-TFSI }\end{array}$} & Pressure $(\mathrm{GPa})$ & 0.0 & 0.4 & 5.6 & 8.1 \\
\hline & Density $\left(\mathrm{g} . \mathrm{cm}^{-3}\right)$ & 1.537 & 1.559 & 1.700 & 1.742 \\
\hline \multirow[t]{2}{*}{ 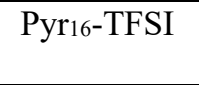 } & Pressure $(\mathrm{GPa})$ & 0.0 & 0.5 & 6.5 & 9.2 \\
\hline & Density $\left(\mathrm{g} . \mathrm{cm}^{-3}\right)$ & 1.321 & 1.348 & 1.501 & 1.540 \\
\hline \multirow{2}{*}{$\begin{array}{c}\text { Pyr16-TFSI + } \\
\text { Li-TFSI } \\
\end{array}$} & Pressure (GPa) & 0.0 & 0.5 & 5.2 & 7.3 \\
\hline & Density $\left(\mathrm{g} \cdot \mathrm{cm}^{-3}\right)$ & 1.442 & 1.472 & 1.599 & 1.635 \\
\hline \multirow[t]{2}{*}{ Pyr $_{19}$-TFSI } & Pressure $(\mathrm{GPa})$ & 0.0 & 0.5 & 4.8 & 8.7 \\
\hline & Density $\left(\mathrm{g} . \mathrm{cm}^{-3}\right)$ & 1.256 & 1.282 & 1.402 & 1.462 \\
\hline \multirow{2}{*}{$\begin{array}{c}\text { Pyr }_{19-T F S I}^{+} \\
\text {Li-TFSI } \\
\end{array}$} & Pressure $(\mathrm{GPa})$ & 0.0 & 0.4 & 2.8 & 6.0 \\
\hline & Density $\left({\left.\mathrm{g} . \mathrm{cm}^{-3}\right)}\right.$ & 1.372 & 1.394 & 1.476 & 1.462 \\
\hline
\end{tabular}

Table S6: Volume occupied by domains formed by TFSI ion at different pressures.

\begin{tabular}{|c|c|c|c|c|c|}
\hline \multirow{2}{*}{$\mathrm{Pyr}_{13}$-TFSI } & Pressure $(\mathrm{GPa})$ & 0.0 & 0.5 & 2.3 & 5.5 \\
\hline & Volume $\left(\mathrm{nm}^{3}\right)$ & 119.04 & 117.06 & 111.92 & 106.89 \\
\hline \multirow{2}{*}{$\begin{array}{c}\text { Pyrr13-TFSI }++ \\
\text { Li-TFSI }\end{array}$} & Pressure $(\mathrm{GPa})$ & 0.0 & 0.4 & 5.6 & 8.1 \\
\hline & Volume $\left(\mathrm{nm}^{3}\right)$ & 113.25 & 111.86 & 103.16 & 100.86 \\
\hline \multirow[t]{2}{*}{ 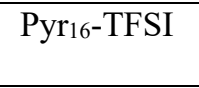 } & Pressure $(\mathrm{GPa})$ & 0.0 & 0.5 & 6.5 & 9.2 \\
\hline & Volume $\left(\mathrm{nm}^{3}\right)$ & 119.86 & 117.28 & 106.16 & 103.66 \\
\hline \multirow{2}{*}{$\begin{array}{c}\text { Pyr }_{16-T F S I}+ \\
\text { Li-TFSI }\end{array}$} & Pressure $(\mathrm{GPa})$ & 0.0 & 0.5 & 5.2 & 7.3 \\
\hline & Volume $\left(\mathrm{nm}^{3}\right)$ & 114.05 & 111.87 & 103.66 & 101.54 \\
\hline \multirow[t]{2}{*}{ Pyr19-TFSI } & Pressure $(\mathrm{GPa})$ & 0.0 & 0.5 & 4.8 & 8.7 \\
\hline & Volume $\left(\mathrm{nm}^{3}\right)$ & 114.91 & 112.24 & 107.84 & 103.00 \\
\hline \multirow{2}{*}{$\begin{array}{c}\text { Pyr }_{19-T F S I}+ \\
\text { Li-TFSI }\end{array}$} & Pressure $(\mathrm{GPa})$ & 0.0 & 0.4 & 2.8 & 6.0 \\
\hline & Volume $\left(\mathrm{nm}^{3}\right)$ & 113.21 & 109.93 & 106.91 & 102.86 \\
\hline
\end{tabular}

Table S7: Volume occupied by single TFSI ion at the highest pressures.

\begin{tabular}{|c|c|c|}
\hline IL & $\begin{array}{l}\text { Volume occupied by cis } \\
\left(\mathrm{cm}^{3} \mathrm{~mol}^{-1}\right)\end{array}$ & $\begin{array}{l}\text { Volume occupied by cis } \\
\qquad\left(\mathrm{cm}^{3} \mathrm{~mol}^{-1}\right)\end{array}$ \\
\hline $\mathrm{Pyr}_{13}$-TFSI & 114.35 & 123.56 \\
\hline $\mathrm{Pyr}_{13}$-TFSI + Li-TFSI & 119.97 & 130.72 \\
\hline Pyr16-TFSI & 117.98 & 135.38 \\
\hline Pyr16-TFSI + Li-TFSI & 118.18 & 134.29 \\
\hline Pyr 19 -TFSI & 117.97 & 132.50 \\
\hline Pyr $_{19}$-TFSI + Li-TFSI & 114.82 & 137.65 \\
\hline
\end{tabular}



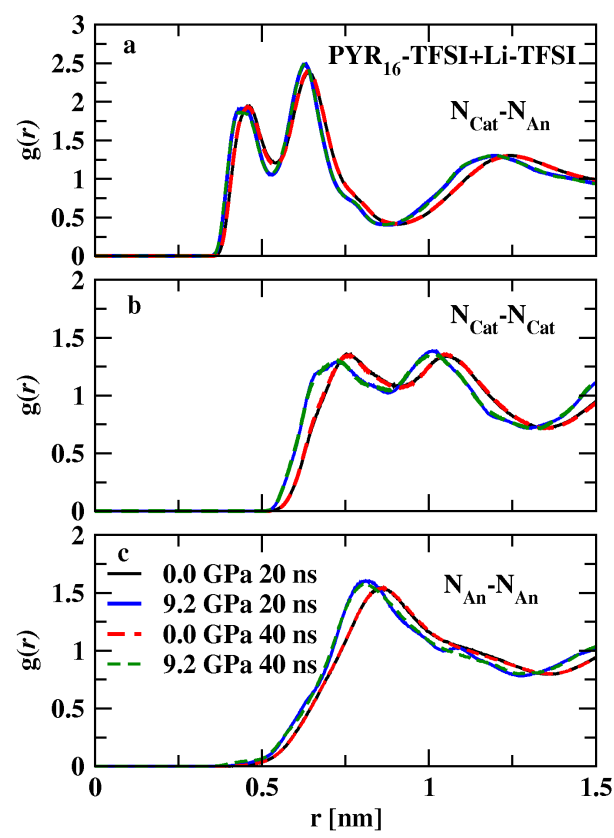

Figure S1: comparison of RDFs from 20 ns production run and 40 ns production run.
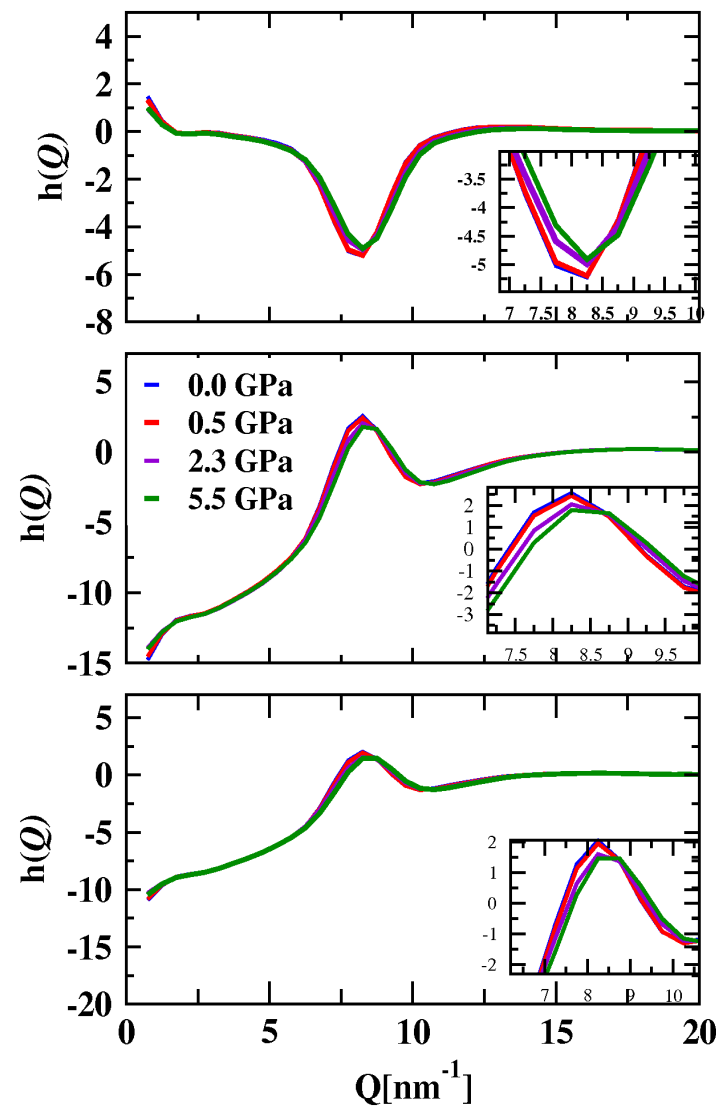

Figure S2: Partial structure factors of cation-anion, cation-cation, and anion-anion of $\mathrm{Pyr}_{13}-\mathrm{TFSI}$ are shown in the top, middle, and bottom rows respectively. 

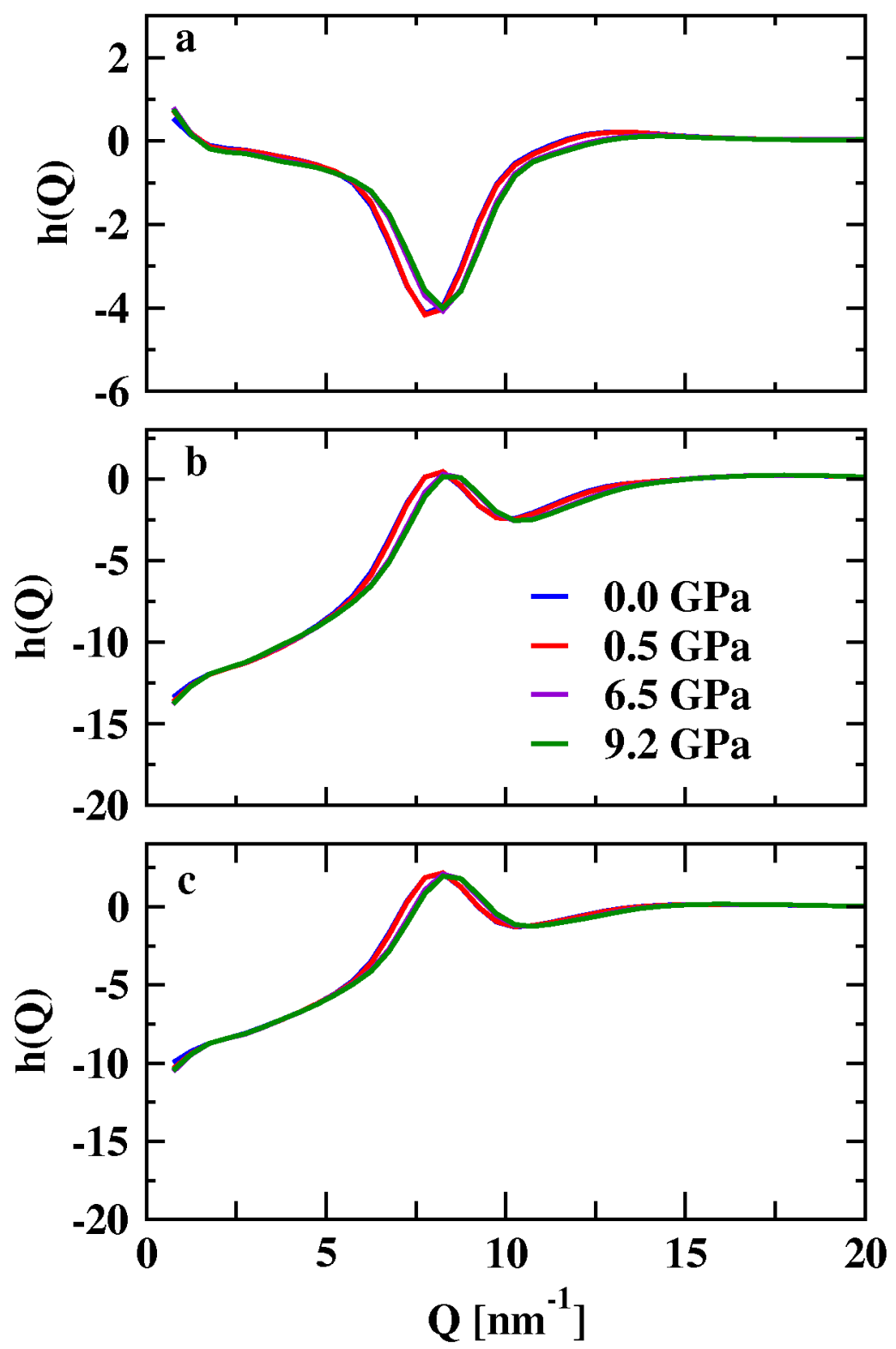

Figure S3: Partial structure factors of cation-anion, cation-cation, and anion-anion of $\operatorname{Pyr}_{16}-\mathrm{TFSI}$ are shown in the a, $\mathrm{b}$, and $\mathrm{c}$. 

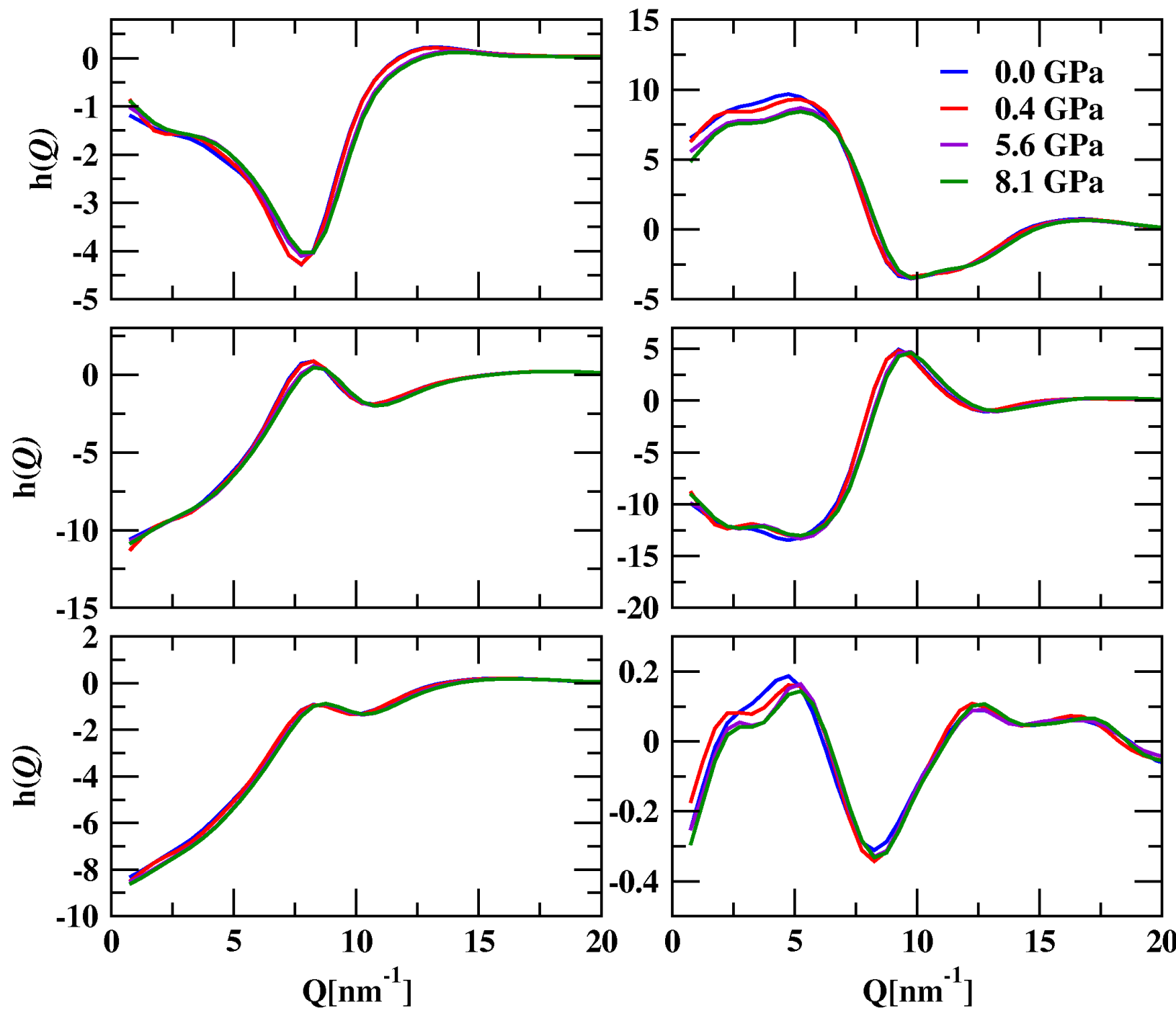

Figure S4: Partial structure functions of $\mathrm{Pyr}_{13}-\mathrm{TFSI}+\mathrm{Li}-\mathrm{TFSI}$ system at different pressures. Cation-anion (left-top), cation-cation (left-middle), anion-anion (left-bottom) Li-anion (right-top), Li-cation (right-middle) and Li-Li (right-bottom). 

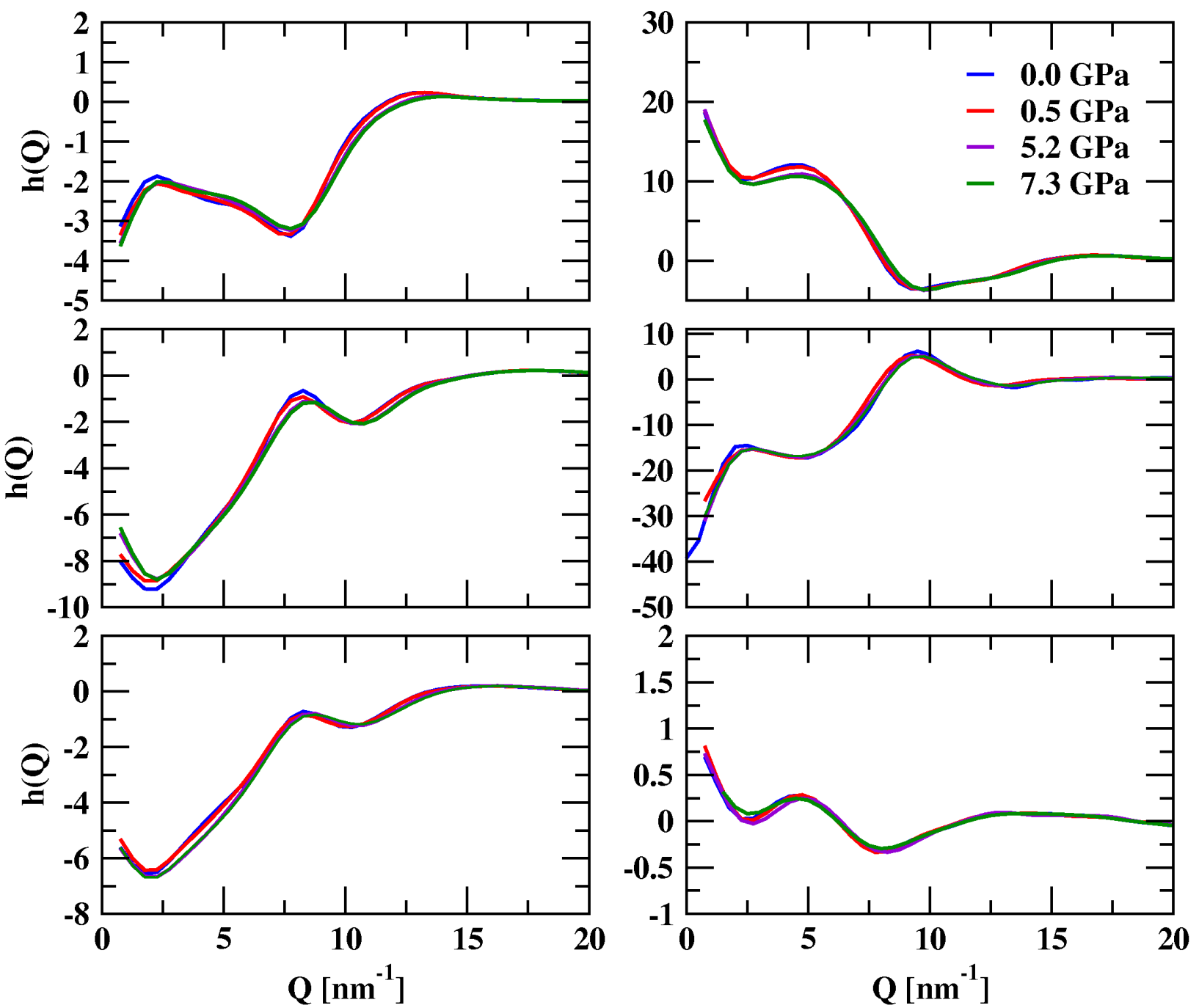

Figure S5: Partial structure functions of $\mathrm{Pyr}_{16}$-TFSI + Li-TFSI system at different pressures. Cation-anion (lefttop), cation-cation (left-middle), anion-anion (left-bottom) Li-anion (right-top), Li-cation (right-middle) and Li-Li (right-bottom). 

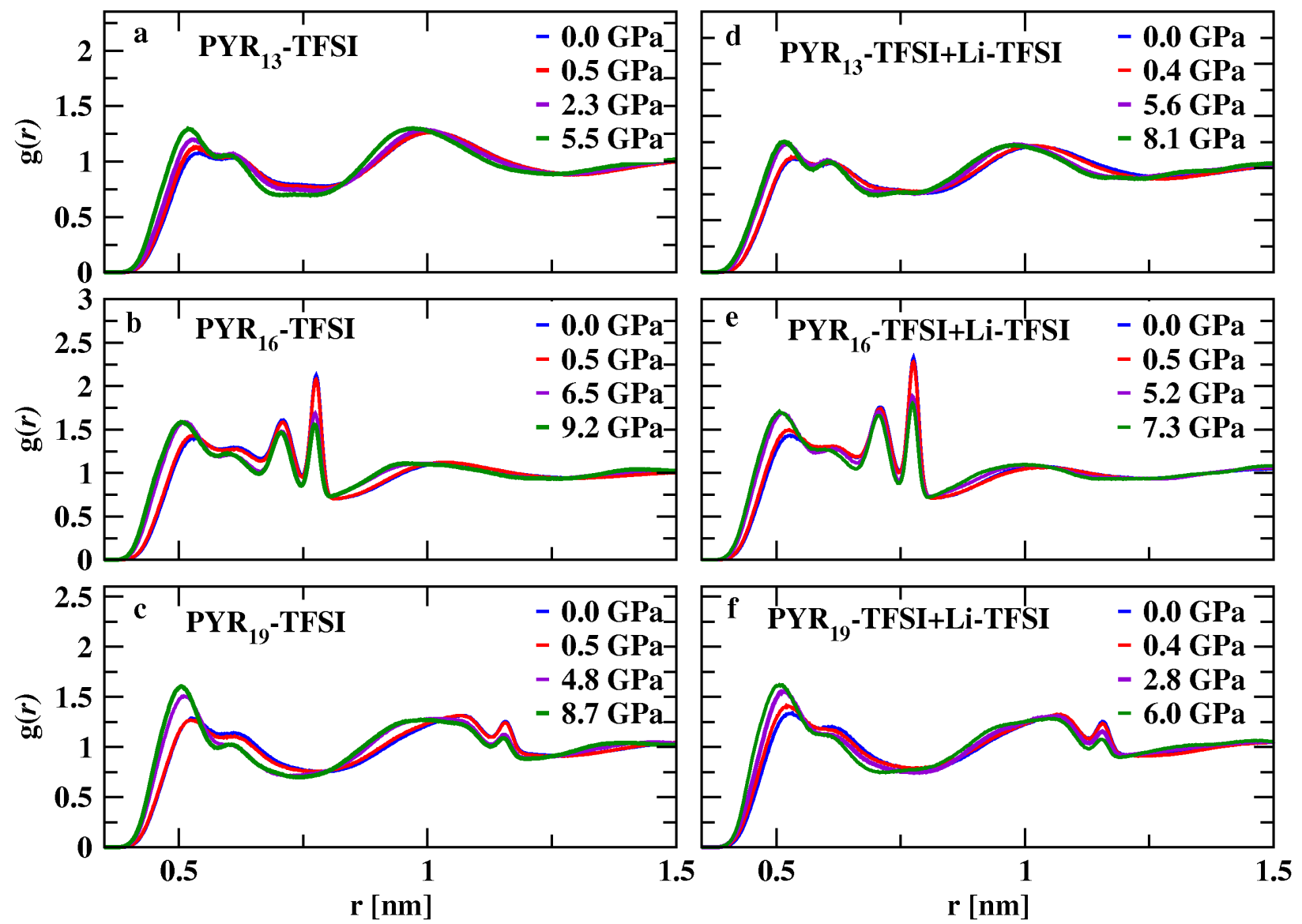

Figure S6: Intermolecular radial distribution function between the nitrogen atom of cation and the terminal carbon atom of the alkyl chain of the cation. 

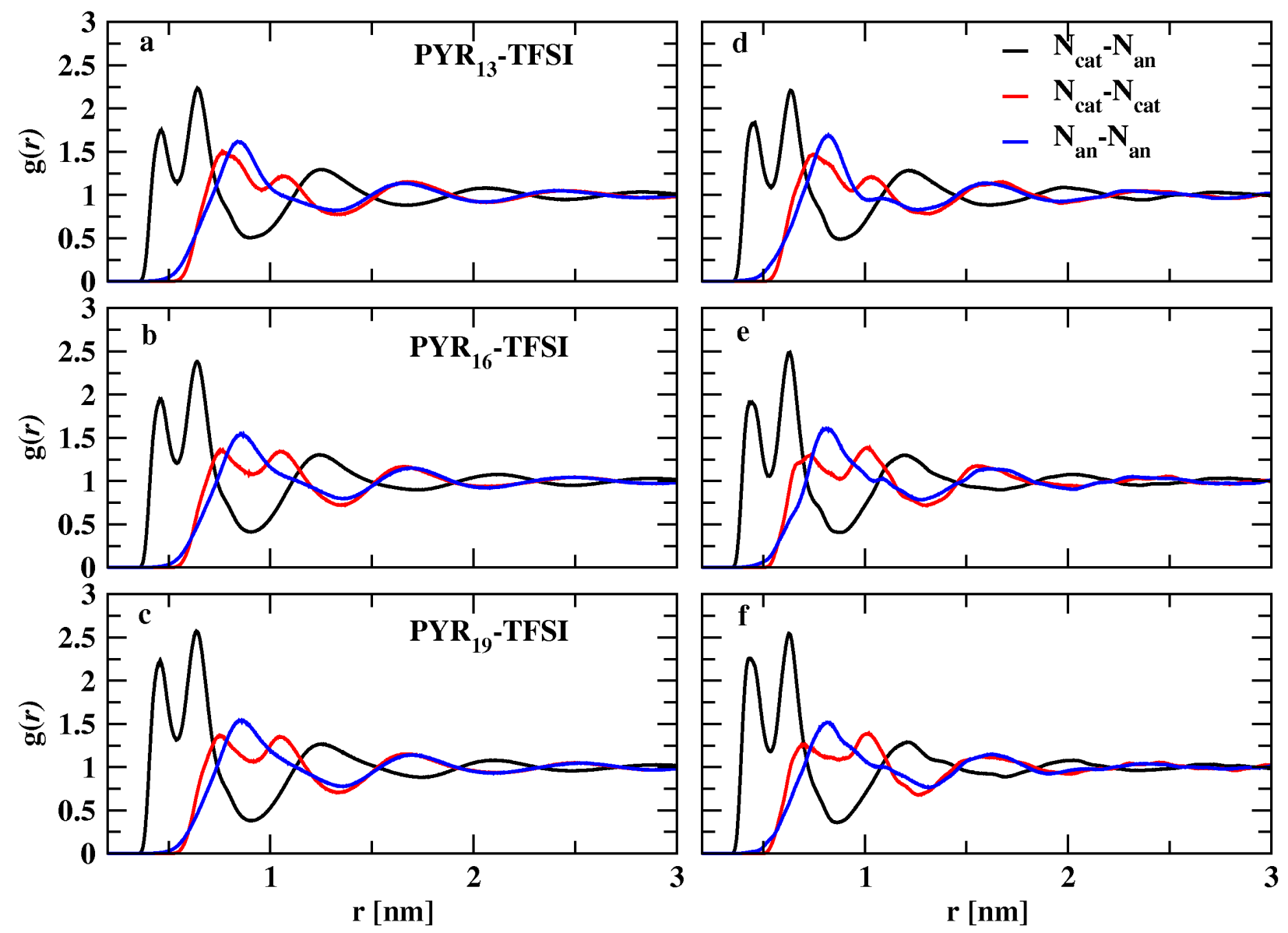

Figure S7: Radial distribution functions between the nitrogen atoms of the cation and the anion of IL. The left column indicates $(a, b, c)$ the lowest pressure and the right column $(\mathrm{d}, \mathrm{e}, \mathrm{f})$ represents the highest pressure. 

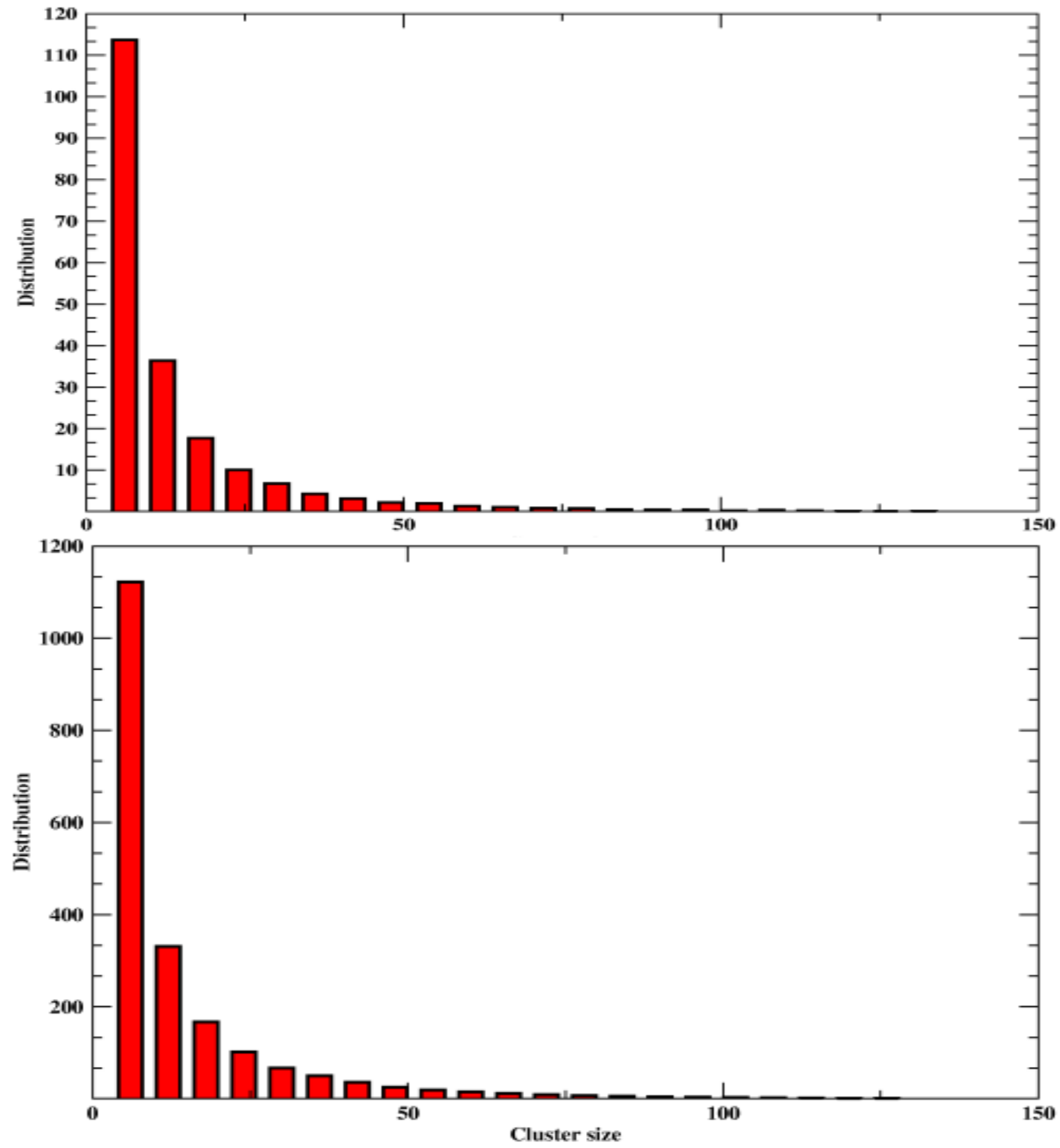

Figure S8: Effect of a number of particles in the simulation box on the distribution of clusters that are formed by alkyl chain in $\mathrm{Pyr}_{16}$-TFSI. The top panel depicts the 500 particles in the simulation box, and bottom panel represents the 5000 particles in the simulation box. 

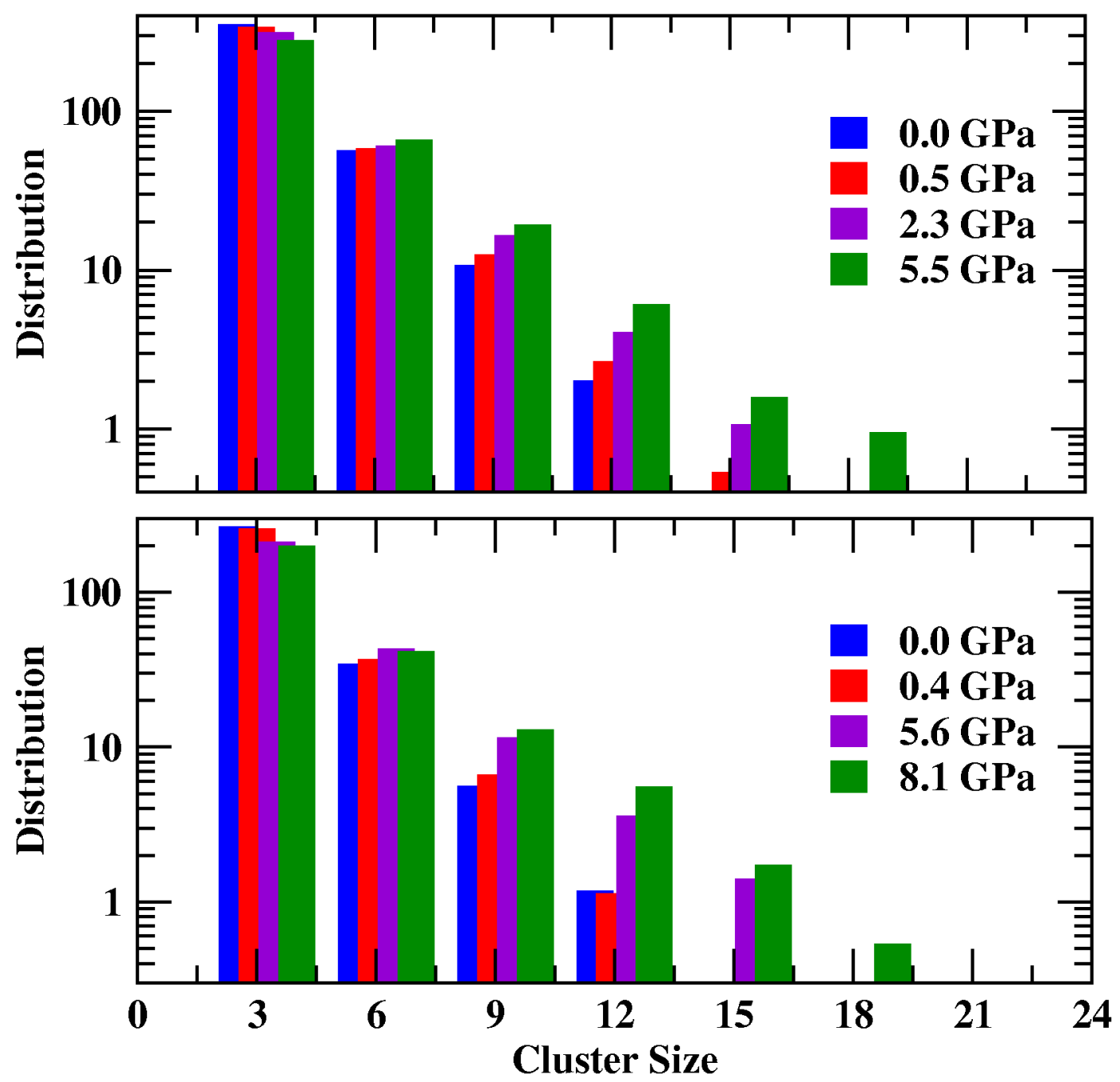

Figure S9: Distribution of clusters that are formed by alkyl chain in $\mathrm{Pyr}_{13}-\mathrm{TFSI}$ (top) and $\mathrm{Pyr}_{13}-\mathrm{TFSI}+\mathrm{Li}-\mathrm{TFSI}$ (bottom) systems. 

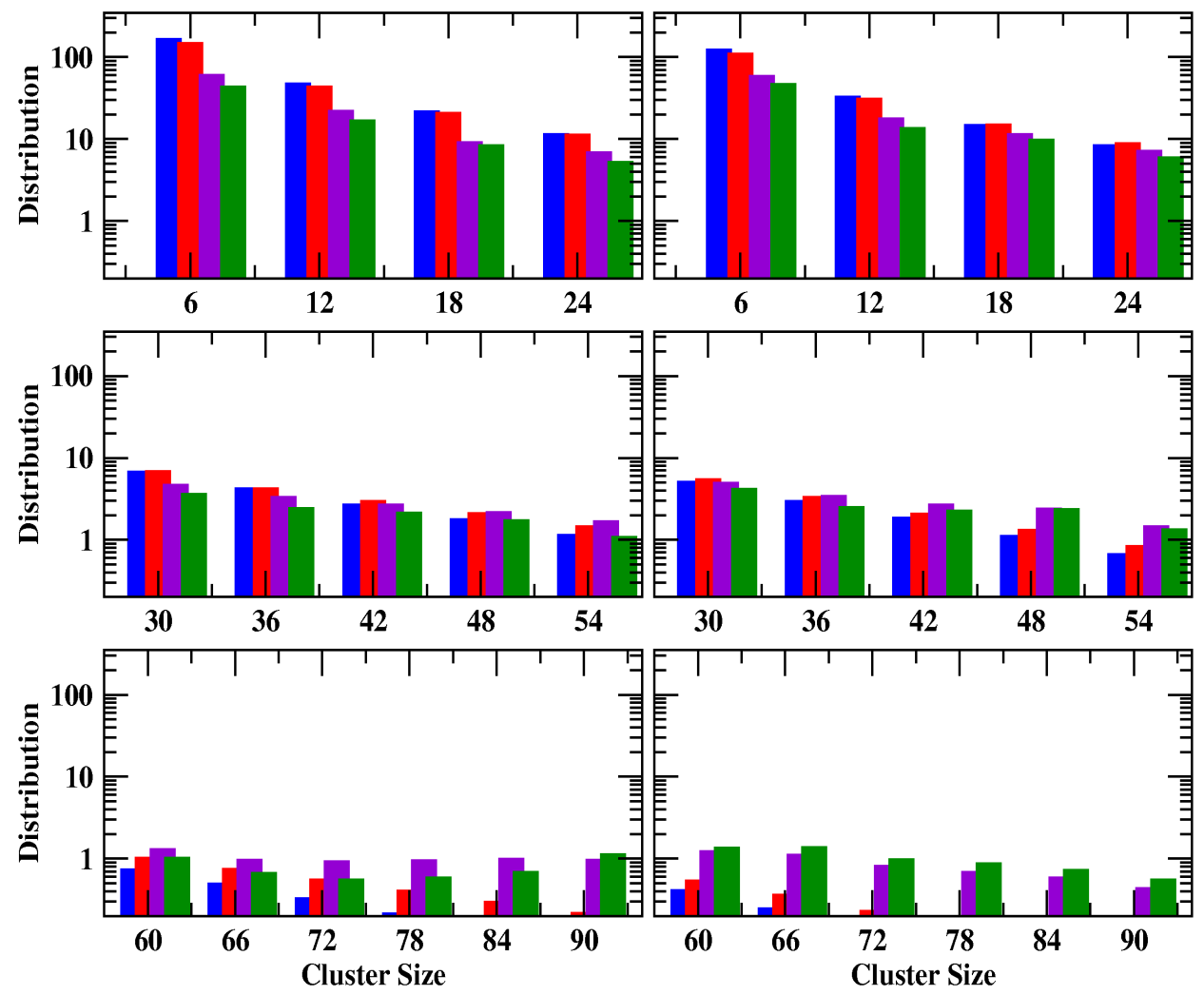

Figure S10: Distribution of clusters that are formed by alkyl chain in $\mathrm{Pyr}_{16}-\mathrm{TFSI}$ (left column) and $\mathrm{Pyr}_{16}-\mathrm{TFSI}+\mathrm{Li}-$ TFSI (right column) systems. 
Optimized structures of ions used in this study.

$\mathrm{PYR}_{13}$
N1 $\quad 0.719 \quad-0.000 \quad 0.077345$
C1 $\quad-0.033 \quad-1.205-0.030255$
$\begin{array}{llll}\text { C2 } & -0.033 & 1.205 & -0.030255\end{array}$
C3 $\quad-1.490 \quad-0.775 \quad-0.028910$
$\begin{array}{llll}\text { C4 } & -1.490 & 0.775 & -0.028910\end{array}$
$\begin{array}{llll}\text { C5 } & 2.141 & -0.000 & -0.435058\end{array}$
$\begin{array}{llll}\text { C6 } & 0.778 & 0.000 & -0.234653\end{array}$
H1 $0.439 \quad-1.441 \quad 0.102369$
H2 $\quad 0.111 \quad-2.048 \quad 0.102369$
H3 $\quad 0.439 \quad 1.441 \quad 0.102369$
$\begin{array}{llll}\mathrm{H} 4 & 0.111 & 2.048 & 0.102369\end{array}$
H5 $\quad-1.871 \quad-1.180 \quad 0.058820$
H6 $\quad-2.125 \quad-1.161 \quad 0.058820$
$\begin{array}{llll}\mathrm{H} 7 & -1.871 & 1.180 & 0.058820\end{array}$
H8 $\quad-2.125 \quad 1.161 \quad 0.058820$
H9 $\quad 2.645 \quad-0.890 \quad 0.193987$
$\begin{array}{llll}\mathrm{H} 10 & 2.645 & 0.890 & 0.193987\end{array}$
$\begin{array}{llll}\mathrm{H} 11 & 2.145 & 0.000 & 0.193987\end{array}$
H12 $1.365 \quad 0.882 \quad 0.138585$
H13 $1.366 \quad-0.881 \quad 0.138585$

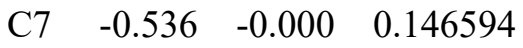
H14 $-1.130 \quad 0.881 \quad 0.028496$
H15 $\quad-1.130 \quad-0.881 \quad 0.028496$
C8 $\quad-0.251 \quad 0.000 \quad-0.301344$
H16 $\quad 0.314 \quad-0.884 \quad 0.101524$
$\begin{array}{llll}\mathrm{H} 17 & 0.314 & 0.884 & 0.101524\end{array}$
$\begin{array}{llll}\mathrm{H} 18 & -1.188 & -0.000 & 0.101524\end{array}$ 

$\mathrm{PYR}_{16}$
N1 $\quad-0.594 \quad-0.000 \quad 0.125579$
C1 $0.286 \quad 1.206 \quad-0.028582$
$\begin{array}{lllll}\text { C2 } & 0.287 & -1.204 & -0.028582\end{array}$
C3 $\quad 1.739 \quad 0.776 \quad-0.038881$
$\begin{array}{lllll}\mathrm{C} 4 & 1.739 & -0.774 & -0.038881\end{array}$
$\begin{array}{llll}\text { C5 } & -1.817 & -0.000 & -0.450610\end{array}$
C6 $\quad-1.088 \quad-0.002 \quad-0.210371$
H1 $0.102 \quad 1.448 \quad 0.097956$
H2 $\quad-0.041 \quad 2.045 \quad 0.097956$
H3 $\quad 0.108 \quad-1.440 \quad 0.097956$
H4 $\quad-0.042 \quad-2.047 \quad 0.097956$
H5 $2.366 \quad 1.178 \quad 0.060871$
H6 $2.126 \quad 1.165 \quad 0.060871$
H7 $\quad 2.372 \quad-1.182 \quad 0.060871$
H8 $\quad 2.118 \quad-1.157 \quad 0.060871$
H9 $\quad-2.406 \quad 0.889 \quad 0.193327$
$\begin{array}{llll}\mathrm{H} 10 & -2.405 & -0.891 & 0.193327\end{array}$
H1 $\quad-1.509 \quad 0.002 \quad 0.193327$
$\begin{array}{llll}\mathrm{H} 12 & -1.726 & -0.883 & 0.122678\end{array}$
H13 $\quad-1.725 \quad 0.880 \quad 0.122678$
$\begin{array}{llll}\text { C7 } & -0.047 & -0.004 & 0.121024\end{array}$
$\begin{array}{llll}\mathrm{H} 14 & 0.591 & -0.888 & 0.012232\end{array}$
$\begin{array}{llll}\text { H15 } & 0.598 & 0.874 & 0.012232\end{array}$
C8 $\quad-0.743 \quad 0.001 \quad-0.037419$
H16 $\quad-1.391 \quad 0.882 \quad 0.017564$
$\begin{array}{llll}\mathrm{H} 17 & -1.400 & -0.872 & 0.017564\end{array}$
C9 $\quad 0.248 \quad-0.002 \quad-0.030207$
$\begin{array}{llll}\mathrm{H} 18 & 0.907 & 0.871 & 0.005266\end{array}$
$\begin{array}{llll}\mathrm{H} 19 & 0.897 & -0.883 & 0.005266\end{array}$
$\begin{array}{llll}\mathrm{C} 10 & -0.436 & 0.003 & 0.167141\end{array}$
$\begin{array}{llll}\mathrm{H} 20 & -1.094 & -0.869 & -0.017198\end{array}$
$\begin{array}{llll}\mathrm{H} 21 & -1.084 & 0.883 & -0.017198\end{array}$ 

$\begin{array}{llll}\text { C11 } & 0.557 & -0.000 & -0.236892\end{array}$
$\begin{array}{llll}\mathrm{H} 22 & 1.206 & 0.879 & 0.063436\end{array}$
$\mathrm{H} 23 \quad 1.196 \quad-0.887 \quad 0.063436$
$\begin{array}{llll}\mathrm{H} 24 & 0.038 & 0.004 & 0.063436\end{array}$

$\mathrm{PYR}_{19}$
$\mathrm{N} 1 \quad-0.561 \quad 0.000 \quad 0.134813$
$\begin{array}{lllll}\text { C1 } & 0.326 & -1.205 & -0.033470\end{array}$
$\begin{array}{llll}\mathrm{C} 2 & 0.326 & 1.205 & -0.033470\end{array}$
C3 $\quad 1.773 \quad-0.775 \quad-0.034491$
$\begin{array}{llll}\text { C4 } & 1.773 & 0.775 & -0.034491\end{array}$
C5 $\quad-1.764 \quad-0.000 \quad-0.459363$
$\begin{array}{llll}\text { C6 } & -1.089 & 0.000 & -0.197979\end{array}$
H1 $\quad 0.168 \quad-1.444 \quad 0.098445$
$\begin{array}{llll}\mathrm{H} 2 & -0.016 & -2.046 & 0.098445\end{array}$
$\begin{array}{llll}\text { H3 } & 0.168 & 1.444 & 0.098445\end{array}$
H4 $\quad-0.016 \quad 2.046 \quad 0.098445$
H5 $\quad 2.421 \quad-1.180 \quad 0.059385$
H6 $2.135 \quad-1.161 \quad 0.059385$
$\begin{array}{llll}\mathrm{H} 7 & 2.421 & 1.180 & 0.059385\end{array}$
$\begin{array}{llll}\mathrm{H} 8 & 2.135 & 1.161 & 0.059385\end{array}$
H9 $\quad-2.357 \quad-0.890 \quad 0.195024$
$\begin{array}{llll}\mathrm{H} 10 & -2.358 & 0.890 & 0.195024\end{array}$
H11 $\quad-1.433 \quad 0.000 \quad 0.195024$
$\begin{array}{llll}\mathrm{H} 12 & -1.728 & 0.882 & 0.119593\end{array}$
$\begin{array}{llll}\mathrm{H} 13 & -1.728 & -0.881 & 0.119593\end{array}$
C7 $\quad-0.073 \quad-0.000 \quad 0.080875$
$\begin{array}{llll}\mathrm{H} 14 & 0.570 & 0.881 & 0.020675\end{array}$
$\begin{array}{llll}\mathrm{H} 15 & 0.570 & -0.882 & 0.020675\end{array}$
C8 $\quad-0.801 \quad-0.000 \quad-0.019264$
$\begin{array}{llll}\text { H16 } & -1.455 & -0.877 & 0.017261\end{array}$ 

$\begin{array}{llll}\mathrm{H} 17 & -1.454 & 0.877 & 0.017261\end{array}$
C9 $\quad 0.164 \quad-0.000 \quad-0.002682$
$\begin{array}{llll}\mathrm{H} 18 & 0.819 & -0.877 & -0.004747\end{array}$
H19 $0.819 \quad 0.876 \quad-0.004747$
$\begin{array}{llll}\mathrm{C} 10 & -0.551 & 0.000 & 0.115654\end{array}$
H20 $\quad-1.207 \quad 0.876 \quad-0.026336$
$\mathrm{H} 21-1.208 \quad-0.876 \quad-0.026336$
$\begin{array}{llll}\text { C11 } & 0.408 & -0.000 & 0.002342\end{array}$
$\begin{array}{llll}\mathrm{H} 22 & 1.064 & -0.877 & -0.011063\end{array}$
$\begin{array}{llll}\mathrm{H} 23 & 1.064 & 0.876 & -0.011063\end{array}$
$\begin{array}{lllll}\mathrm{C} 12 & -0.305 & 0.000 & -0.005032\end{array}$
$\mathrm{H} 24 \quad-0.962 \quad 0.876 \quad-0.004126$
$\mathrm{H} 25 \quad-0.962 \quad-0.876 \quad-0.004126$
$\begin{array}{lllll}\mathrm{C} 13 & 0.652 & -0.000 & 0.161433\end{array}$
H26 $\quad 1.308 \quad-0.876 \quad-0.025336$
$\begin{array}{llll}\mathrm{H} 27 & 1.308 & 0.875 & -0.025336\end{array}$
$\begin{array}{llll}\mathrm{C} 14 & -0.068 & 0.000 & -0.224180\end{array}$
$\begin{array}{llll}\mathrm{H} 28 & 0.642 & 0.000 & 0.053688\end{array}$
$\begin{array}{llll}\mathrm{H} 29 & -0.705 & 0.883 & 0.053688\end{array}$
$\begin{array}{llll}\mathrm{H} 30 & -0.705 & -0.882 & 0.053688\end{array}$

TFSI

N1 $\quad-0.002 \quad-0.808 \quad-0.638984$

S1 $\quad 0.852 \quad-0.070 \quad 0.998272$

S2 $\quad-0.853 \quad-0.070 \quad 0.998272$ 


$\begin{array}{llll}\text { O1 } & -1.850 & -1.001 & -0.528979 \\ \text { O2 } & -1.227 & 1.308 & -0.528979 \\ \text { O3 } & 1.226 & 1.308 & -0.528979 \\ \text { O4 } & 1.849 & -1.001 & -0.528979 \\ \text { C1 } & 0.369 & 0.023 & 0.383018 \\ \text { C2 } & -0.368 & 0.023 & 0.383018 \\ \text { F1 } & -0.229 & 0.582 & -0.167947 \\ \text { F2 } & 0.795 & -1.196 & -0.167947 \\ \text { F3 } & 1.439 & 0.764 & -0.167947 \\ \text { F4 } & 0.231 & 0.583 & -0.167947 \\ \text { F5 } & -0.793 & -1.196 & -0.167947 \\ \text { F6 } & -1.439 & 0.764 & -0.167947\end{array}$

